\section{Humbert Lesca, \\ Veille stratégique : la méthode L.E.SCAnnig, Colombelles, Éditions EMS, 2003, 190 p.}

Un livre pas comme les autres et qui ne se lit pas comme les autres ce qui, bien sûr, ne minimise pas son intérêt. Pour en avoir une idée immédiate, rien de plus facile, car l'auteur, dans les premières lignes de l'introduction, invite le lecteur à aller surfer sur : www.veille-stratégique.org. On y trouve de larges extraits du premier chapitre dont toutes les définitions essentielles pour la bonne compréhension de l'ensemble de l'ouvrage qui comprend dix chapitres. On devine rapidement que la méthode ainsi nommée se veut un jeu de mots, à mon avis pas très heureux, avec le patronyme de l'auteur. Est-il nécessaire de signaler que "scanning » est la traduction en langage shakespearien de ce que Molière qualifiait de « veille». Humbert Lesca, en plus d'avoir en commun avec moi un intérêt particulier envers le Brésil (cf le site Web en question), est docteur en gestion et enseigne à l'Université Pierre-Mendès-France de Grenoble et dirige une équipe de recherche depuis une quinzaine d'années dont l'ouvrage présente en quelque sorte une synthèse des travaux réalisés.

La définition du mot veille donne une idée du style bien vivant qui caractérise ce volume. Qu'on en juge : « Ce mot est à prendre dans son sens exact et qui signifie être éveillé (le contraire d'endormi !), être en état de réceptivité, être prêt à détecter quelque chose qui pourrait se produire sans que l'on sache exactement quoi ni où. L'attention est au repos mais prête à se déclencher à la moindre alerte. Dans le contexte de notre méthode, il s'agit de veille à l'égard de l'environnement extérieur à l'entreprise (ou autre organisation) ». Un nota bene indique qu'il ne faut pas confondre: «Veille Anticipative Stratégique» avec «Veille Technologique», cette dernière ne concernant qu'un aspect particulier de l'environnement de l'entreprise. En revanche, la Veille Stratégique englobe aussi la veille concurrentielle, commerciale, les fournisseurs, etc. Et il est beaucoup question de la VAS-IC dont j'ai cherché en vain une définition. Mais on nous indique que sa finalité est de permettre à l'entreprise d'agir vite, au bon moment, avec le maximum d'efficacité et le minimum de ressources, dans le but de contribuer à sa compétitivité durable. Il importe de savoir que la VAS-IC ne concerne pas que les entreprises, elle peut également être utile pour toutes sortes d'organisations. Avis aux intéressés, donc !

La forme se veut conforme au style. Ce n'est pas un ouvrage pour les amoureux de la littérature. Inutile de penser le lire d'un trait et le ranger ensuite dans une oubliette quelconque. Il est fait, au contraire, pour être consulté suivant les besoins. On y trouve une foule de graphiques, schémas, encadrés, exemples de questions et autres tableaux. Tout ceci en vue d'aider la prise de décision, que ce soit au sein d'une entreprise ou de tout organisme. Bon amusement !

\section{Guy Loinger (dir.), La prospective régionale, de chemins en desseins, Paris, Les Éditions de l'Aube, DATAR, 2004, 275 p.}

En sa qualité de responsable depuis des années de l'Observatoire international de prospective rattaché à l'Université de Paris I, Guy Loinger, au début de 2003, m'a invité à collaborer à ce volume en espérant obtenir la description d'une région québécoise nantie d'une expérience de prospective territoriale. Considérant qu'il n'en existait pas, j'avais décliné l'invitation. Quelques mois plus tard, j'apprenais que des collègues du réseau uquiste, ne partageant de toute évidence pas mon point de vue, se sont engagés à fournir un exemple québécois. C'est donc avec empressement que j'ai cherché cet exemple en recevant l'ouvrage. En vain. Les collègues se sont soit ralliés à mon point de vue ou soit, plus vraisemblablement, n'ont pas pris le temps de remplir leur engagement. À la lecture de ce qui suit, il est possible de juger si, à l'époque du moins, j'avais raison.

Malgré son titre, l'ouvrage traite de prospective territoriale. Il revient au lecteur de distinguer une région d'un territoire. Dans sa préface, le prolifique Michel Gaudet signale que c'est une mauvaise idée que de vouloir en imposer une bonne. Le ton est ainsi donné. On comprend immédiatement que la prospective territoriale se fait à plusieurs, la concertation étant le mot clé. Si elle fait appel à la démarche des scénarios, le résultat, signale Godet, est moins important que le processus collectif qui y a conduit. Faut se parler! disait ce fameux slogan publicitaire québécois du début des années 1980. Et c'est ce dont l'ouvrage veut témoigner à partir des exemples suivants où les 